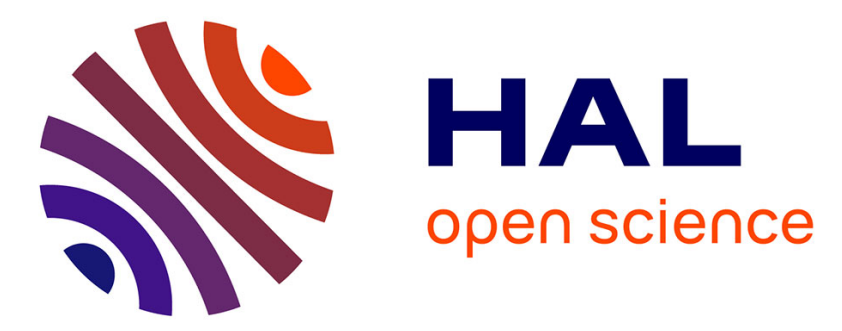

\title{
Bloch surface waves at the telecommunication wavelength with lithium niobate as the top layer for integrated optics
}

Tatiana Kovalevich, Djaffar Belharet, Laurent Robert, Gwenn Ulliac, Myun Kim, Hans Peter Herzig, Thierry Grosjean, Maria Artajona

\section{To cite this version:}

Tatiana Kovalevich, Djaffar Belharet, Laurent Robert, Gwenn Ulliac, Myun Kim, et al.. Bloch surface waves at the telecommunication wavelength with lithium niobate as the top layer for integrated optics. Applied Optics, 2019, 58 (7), pp.1757 - 1762. 10.1364/ao.XX.XXXXXX . hal-02371031

\section{HAL Id: hal-02371031 \\ https://hal.science/hal-02371031}

Submitted on 1 Dec 2020

HAL is a multi-disciplinary open access archive for the deposit and dissemination of scientific research documents, whether they are published or not. The documents may come from teaching and research institutions in France or abroad, or from public or private research centers.
L'archive ouverte pluridisciplinaire HAL, est destinée au dépôt et à la diffusion de documents scientifiques de niveau recherche, publiés ou non, émanant des établissements d'enseignement et de recherche français ou étrangers, des laboratoires publics ou privés. 


\title{
Bloch surface waves at the telecommunication wavelength with Lithium Niobate as top layer for integrated optics.
}

\author{
Tatiana Kovalevich ${ }^{1, *}$, Djaffar Belharet ${ }^{1}$, Laurent Robert ${ }^{1}$, Gwenn Ulliac ${ }^{1}$, Myun-Sik \\ Kim $^{2}$, Hans Peter Herzig ${ }^{2}$, Thierry Grosjean ${ }^{1}$, and Maria-Pilar Bernal ${ }^{1}$ \\ ${ }_{1}^{1}$ Département d'Optique P.M. Duffieux, Institut FEMTO-ST, UMR 6174 CNRS, Université Bourgogne Franche-Comté, 15B Avenue des Montboucons, Besançon \\ Cedex, France, 25030 \\ ${ }^{2}$ Optics \& Photonics Technology Laboratory, Ecole Polytechnique Fédérale de Lausanne (EPFL), Rue de la Maladière 71b, Neuchâtel, Switzerland, CH-2000 \\ Corresponding author: tnkovalevich@gmail.com \\ Compiled December 12, 2018
}

Lithium niobate (LN) based devices are widely used in integrated and nonlinear optics. This material is robust and resistive to high temperatures, which makes the LN-based devices stable, but challenging to fabricate. In this work we report on the design, manufacturing and characterization of engineered dielectric media with thin film lithium niobate (TFLN) on top for the coupling and propagation of electromagnetic surface waves at the telecommunication wavelengths. The designed one-dimentional photonic crystal (1DPhC) sustains Bloch surface waves at the multilayer/air interface at $1550 \mathrm{~nm}$ wavelength with a propagation detected over a distance of $3 \mathrm{~mm}$. The working wavelength and improved BSW propagation parameters open the way for exploration of nonlinear properties of BSW based devices. It is also expected that these novel devices potentially would be able to modify BSW propagation and coupling by external thermal/electrical stimuli due to the improved quality of the TFLN top layer of 1DPhC. () 2018 Optical Society of America

http://dx.doi.org/10.1364/ao.XX.XXXXXX

\section{INTRODUCTION}

Bloch surface waves (BSWs) [1] are electromagnetic surface waves excited at the interface between a truncated periodic dielectric multilayer and a surrounding medium. Bloch surface waves (BSWs) have been studied extensively and proved to be efficient in biosensing[2-10], optical trapping[11], vapor sensing[12] and fluorescent enhancement [13, 14]. BSWs have been recently used to study third harmonic generation in thin film of GaAs[15], thus exploiting nonlinear applications. Moreover, the propagation length[16] and dynamics[17] of surface plasmons is also improved using BSWs.

Due to long propagation length and strong field confinement BSWs find applications in the field of integrated op- tics, thanks to low loss dielectric materials[18]. The fundamental dielectric 2D optical elements, such as 2D-lenses and sub-wavelength focusers[19, 20], ridge waveguides[21-23], 2D prisms, gratings[24] and theoretical resonators[25, 26] have been studied to manipulate the propagation of BSWs. The results have proven BSWs as a novel candidate for 2D optics. Further, R. Dubey et. al.[27] have studied thoroughly more complex ultra-thin optical components which are the key elements of integrated optics applications. These compounds include 2D disk resonators[28-31], BSWs reflectors[32], 2D non-diffracting beam[30] and graphene based in-plane photodetectors[33]. The above listed studies contribute to the significant advancement of BSWs based 2D optical integrated systems.

With the aim of finding new optical functionalities various materials have been used for multilayers and the top (device) layer. For example $\mathrm{Ta}_{2} \mathrm{O}_{5} / \mathrm{SiO}_{2}[10,34], \mathrm{SiO}_{2} / \mathrm{Si}_{3} \mathrm{~N}_{4}$ [19], $\mathrm{TiO}_{2}$ [18], graphene [33, 35], ZnS [36], $\mathrm{MoS}_{2}$ [37] and $\mathrm{LiNbO}_{3}$ [38].

Lithium niobate $\left(\mathrm{LiNbO}_{3}\right)$ is a high refractive index birefringent nonlinear material which has been widely used in integrated optics. Lithium niobate based sensors[39, 40], modulators[41], ring and disk resonators[39, 42] have been studied, thanks to its ferro-electrical, piezo-electrical, thermoelectrical properties and transparency over a wide wavelength range $(350 \mathrm{~nm}-5200 \mathrm{~nm})$. Being a top layer in many devices, $\mathrm{LiNbO}_{3}$ provides an enhanced field confinement[39], which can be improved even more by the use of thin films[40].

Recently this material was introduced as a top layer of BSWs based one-dimensional photonic crystal (1DPhC) structures[43] It has been demonstrated experimentally that modifiable properties of one layer would bring a tunability to the whole 1DPhC[38]. Thus one can expect a future possibility to induce nonlinear behavior and electro/thermo- modulation of BSWs using TFLN as a top layer of the $1 \mathrm{DPhC}$ structure.

In our previous works we proved possible to use $\mathrm{LiNbO}_{3}$ as a part of a multilayer structure. Yet, as previously reported, $1 \mathrm{DPhCs}$ have several limitations. At first, TFLN was embedded into the multilayer stuck[38], which limited the use of BSWs for any sensing applications and made impossible TFLN nanostructuring. Later, several fabrication protocols were developed 


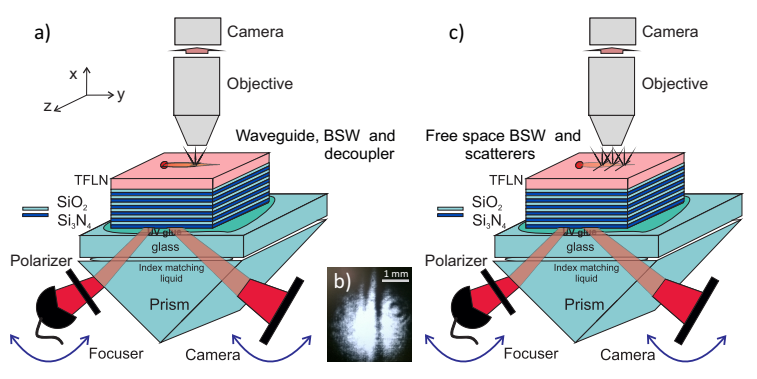

Fig. 1. (a) Experimental setup for the BSW propagation in the waveguide, (b) bottom camera image, (c) experimental setup for a free-space BSW decoupling on scatterers.

in order to bring TFLN to the top of 1DPhC[43]. Following these fabrication protocols BSWs in the visible part of spectrum were exited. Unfortunately, these protocols did not allow to achieve high quality of the top TFLN surface, what prohibited the BSW propagation on longer wavelengths.

In this paper, we develop a new fabrication process and investigate the coupling and propagation of BSWs on a thin film $\mathrm{LiNbO}_{3}$ based $1 \mathrm{DPhC}$ system in order to develop 2D optical systems at telecommunication wavelengths with potential tunability.

\section{MODELING}

Our 1DPhC design consists of four pairs of $\mathrm{SiO}_{2}$ and $\mathrm{Si}_{3} \mathrm{~N}_{4}$ with $450 \mathrm{~nm}$ thick X-cut TFLN on the top (Fig. 1a,c). The design is optimized in order to work at the telecommunication wavelength of $1550 \mathrm{~nm}$ for the applications in integrated optics.

In order to create a multilayer structure, we select a thickness of TFLN, which is commercially available and close to fulfill the Bragg condition. Then, we optimize the thicknesses and number of layers via impedance approach[44] for transverse electric (TE) incidence light.

In order to achieve the maximum coupling of surface waves, the thicknesses of $\mathrm{Si}_{3} \mathrm{~N}_{4}$ and $\mathrm{SiO}_{2}$ are optimized according to the maximum field extinction ratio (E.R.) for one pair. The details of the maximum field extinction ratio method can be found in ref. [44]. The optimized thicknesses are $250 \mathrm{~nm}$ and 450 $\mathrm{nm}$ for $\mathrm{Si}_{3} \mathrm{~N}_{4}$ and $\mathrm{SiO}_{2}$ respectively. The numerical solution for the E.R. at wavelength $1550 \mathrm{~nm}$, with refractive indices of $\mathrm{n}_{\mathrm{Si}_{3} \mathrm{~N}_{4}}=1.79+0.001 i$ and $\mathrm{n}_{\mathrm{SiO}_{2}}=1.44+0.001 i$, is shown in the Fig. (2a).

Pockels effect is a commonly used phenomena for the refractive index change in crystals that lack inversion symmetry, such as $\mathrm{LiNbO}_{3}[45]$. For our optimized multilayer design we chose the configuration which can potentially utilize the largest electro-optic coefficient, and hence leads to a higher change of the refractive index. For $\mathrm{LiNbO}_{3}$ it corresponds to the coefficient $r_{33}=30.8 \mathrm{pm} / \mathrm{V}$ at the wavelength of $1550 \mathrm{~nm}$. In this case, for $\mathrm{X}$-cut TFLN, the electrodes should be placed along $\mathrm{Y}$ axis of the $\mathrm{LiNbO}_{3}$. Therefore, BSWs should propagate along the $\mathrm{Y}$ direction. For the multilayer design modeling we take the ordinary refractive index of $\mathrm{LiNbO}_{3} n_{0}=2.199+0.001 i$. For the TFLN the refractive index $\mathrm{n}_{\mathrm{LiNbO}_{3}}$ is obtained from ellipsometry measurements [38]. For multilayer materials, the imaginary part $\left(\mathrm{n}_{i}\right.$ $=0.001 \mathrm{i}$ ) of refractive indices is introduced to consider the losses at the layer interfaces and to locate the reflection dip[46].

We exploit Kretschmann configuration[47] to couple BSWs
(Fig. 1a,c). The coupling of surface waves depends on the total extinction in the layers and can be verified by observing the reflection dip[44]. For TE polarized incident light, the reflectance dip can be observed at $49^{\circ}$ with reflectance dumping for $70 \%$, see Fig. (2b).

For the presented multilayer design, the dispersion curve for $1 \mathrm{DPhC}$ is shown in Fig. (2c). The external medium is air. We take into consideration the non-homogeneity of the TFLN layer on the three-inch wafer in which it is purchased. This nonhomogeneity appears due to fabrication process of TFLN[49]. The thickness of TFLN layer varies within $50 \mathrm{~nm}$ (from 440 $\mathrm{nm}$ to $490 \mathrm{~nm}$ ) at different positions on the wafer as a defect during fabrication. Considering the thickness variation of the TFLN layer, BSWs can still be excited on 1DPhC stack with aforementioned thicknesses, but at a slightly different incident angle. The dispersion curve of BSWs obtained via impedance approach, in Fig. (2c), would still remain within the photonic band gap of the $1 \mathrm{DPhC}$ with a slight shift in the position. The position of the dispersion curve inside the band gap indicates that BSWs cannot propagate in multilayers because of the fact that the photonic band gap and total internal reflection on the other side restricts the BSW propagation in an external medium (air in our case). Therefore, light tightly remains confined to the interface of the $1 \mathrm{DPhC}$ and the external medium.

\section{FABRICATION}

In our previous work [43], we demonstrated several fabrication approaches in order to release the top TFLN surface for external medium sensing and additional nano-structuring, which is essential for integrated optics. Several membrane-based and onglass-support 1DPhC configurations were proposed in order to excite BSWs at the TFLN/air interface at a wavelength of $473 \mathrm{~nm}$. The samples fabricated on the base of a suspended membrane are fragile and not very comfortable to operate in Kretschmann configuration.

In this study, we focus on on-glass-support 1DPhC configuration, where a larger area of 1DPhC with TFLN layer is available for BSWs manipulation. The robustness of this configuration facilitates the experimental conditions. By following the fabrication steps described in [43] one indeed can obtain a 1DPhC which sustains a BSW in the visible part of the spectrum. However, these $1 \mathrm{DPhCs}$ do not support surface waves at higher wavelengths, particularly at the telecommunication wavelength $1550 \mathrm{~nm}$.

After SEM (FEI, Quanta 450W) investigation of the top surface of fabricated 1DPhC as discussed in [43], some residuals of $\mathrm{SiO}_{2}$ were detected (see Fig. 3a). The size of the surface defects is such that the propagation of the BSWs on TFLN surface is possible in the visible part of spectrum, however surface imperfections do not allow surface wave propagation at the desirable wavelength of $1550 \mathrm{~nm}$. These surface imperfections are removed by extra cleaning in piranha solution between fabrication steps and by significant modifications in $\mathrm{Si}$ and $\mathrm{SiO}_{2}$ process. The SEM image of TFLN surface of 1DPhC fabricated following the new protocols is shown in Fig. 3b. The fabrication process is discussed in the following paragraphs.

The surface wave existence imposes the requirement of thin layers on the top of $1 \mathrm{DPhC}$. In case of lithium niobate, the deposition of thin layers is challenging. This is because lithium niobate needs to maintain the crystallinity in order to be utilized as a nonlinear material. Nowadays technologies such as sputtering, evaporation or epitaxial growth allow only amorphous 

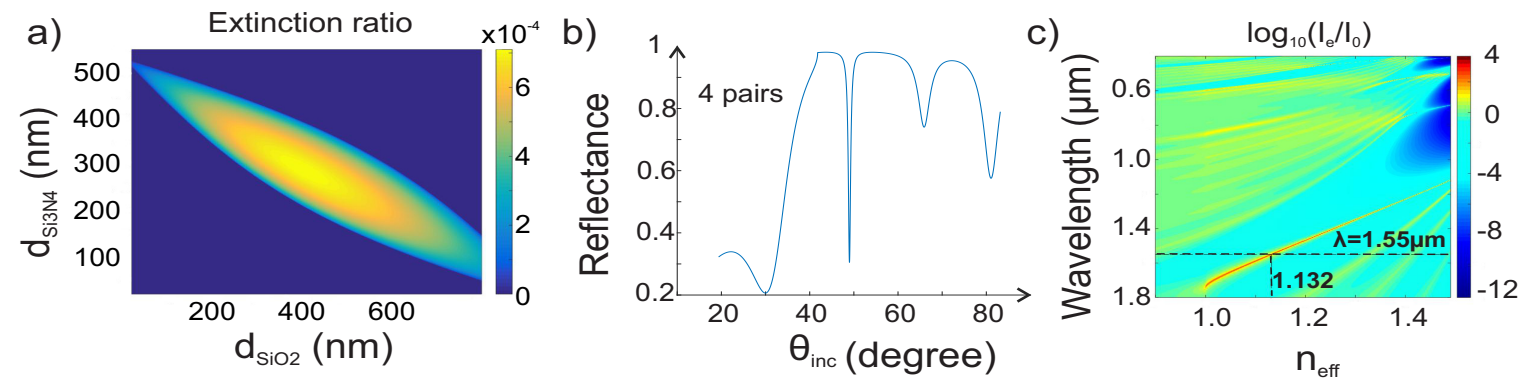

Fig. 2. (a) Extinction of the band gap as a function of layer thicknesses in $1 \mathrm{DPhC}$, (b) Calculated reflectance at $1550 \mathrm{~nm}$ wavelength for different incidence angles, (c) Dispersion curve of the 1DPhC. The modeling was performed for TE polarized incident light for BK7 glass/ $\left(\mathrm{Si}_{3} \mathrm{~N}_{4}-250 \mathrm{~nm} / \mathrm{SiO}_{2}\right) \times 4 / \mathrm{TFLN}-450 \mathrm{~nm} /$ air stuck. $I_{e} / I_{0}$ is a ratio of the light intensity at TFLN/air interface and the intensity of the incident light. $n_{\text {eff }}$ is the effective refractive index of BSW.

or polycrystalline film deposition of $\mathrm{LiNbO}_{3}$ [48]. For sample fabrication, a single-crystal TFLN $(450 \mathrm{~nm})$ bonded to a $\mathrm{SiO}_{2}$ layer on Si substrate [49] is used.

The TFLN is prepared using a $\mathrm{He}+$ ion implantation technique[49]. The prepared sample is cleaned with acetone, ethanol and piranha solution. The multilayer stack of alternating $\mathrm{SiO}_{2}$ and $\mathrm{Si}_{3} \mathrm{~N}_{4}$ are deposited on TFLN by PECVD (Oxford Plasmalab80+). The whole structure is bonded to the glass holder $(500 \mu \mathrm{m})$ by UV glue and cleaned once again in acetone, ethanol and piranha. The optical matching between the multilayer and the glass holder is insured by UV glue (VITRALIT 6127), which is especially designed for glass bonding. The whole stack is protected by $\mathrm{S} 1813$ photoresist, and $500 \mu \mathrm{m}$ of $\mathrm{Si}$ is dry etched by deep reactive ion etching (DRIE). For this etching, the Bosch process $[50,51]$ with the end point detection is employed with $\mathrm{SiO}_{2}$ as a stop layer. The end point detection system based on OES Optical emission spectroscopy (OES) was used in order to achieve precise etching of silicon when reaching the $\mathrm{SiO}_{2}$ stop layer. Afterwards, the $2 \mu \mathrm{m}$ thickness layer of $\mathrm{SiO}_{2}$ is etched by wet etching in a $40 \%$ HF solution.

Cleaning in the piranha solution allows to avoid organic contamination and, as a consequence, the micro-masking effect during DRIE. The cleaning should be performed on the sample without phtoresist or UV glue. The end point detection for Si etching allows to avoid over-etching and optimizes the fabrication time. Final wet etching of $\mathrm{SiO}_{2}$ insures homogeneous removal of this layer together with some residuals of Si left after DRIE.

As shown in Fig. 3b, a drastic surface improvement is
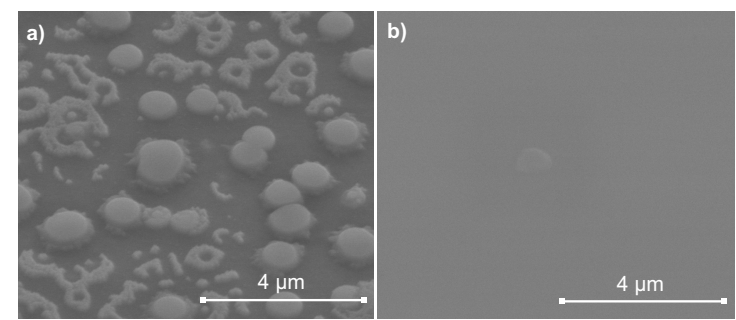

Fig. 3. SEM (FEI, Quanta 450W) images of 1DPhC with TFLN top surface before (a) and after (b) implementation of additional cleaning and temperature stabilization protocols. achieved after implementing the above mentioned fabrication steps. This allows the structuring of the top layer of LN to pattern micro optical elements into it. The cross-section of the fabricated sample is shown in Fig. 4a. In order to obtain the SEM image a small opening on the top surface is made by FIB milling. In order to avoid charging effect, an additional layer of $200 \mathrm{~nm}$ of $\mathrm{Cr}$ is deposited by sputtering. After FIB milling and sample characterization, $\mathrm{Cr}$ is removed by a $\mathrm{Cr}$-etch solution.

Figure $4 \mathrm{~b}$ shows a part of the $200 \mu \mathrm{m}$ long and $12 \mu \mathrm{m}$ wide BSW ridge multimode waveguide. The waveguide is milled by FIB (FEI Helios 600i) milling along Y crystalline axis of $\mathrm{LiNbO}_{3}$. Thus, it would be possible to exploit the largest electro-optical coefficient of lithium niobate (e.g. by depositing electrodes along $\mathrm{Y}$ axis from the both sides of the waveguide). The waveguide is composed of two grooves of $1 \mu \mathrm{m}$ depth and $1 \mu \mathrm{m}$ width, respectively. At the end of the waveguide, gratings are milled to perform as a decoupler at $1550 \mathrm{~nm}$ wavelength. The grating consists of ten periodic grooves of $1 \mu \mathrm{m}$ depth and $0.7 \mu \mathrm{m}$ width. The period of the grating is $1.4 \mu \mathrm{m}$.

Additionally, as a separate experiment, for the BSW propagation estimation, scatterers are fabricated on the top of the 1DPhC
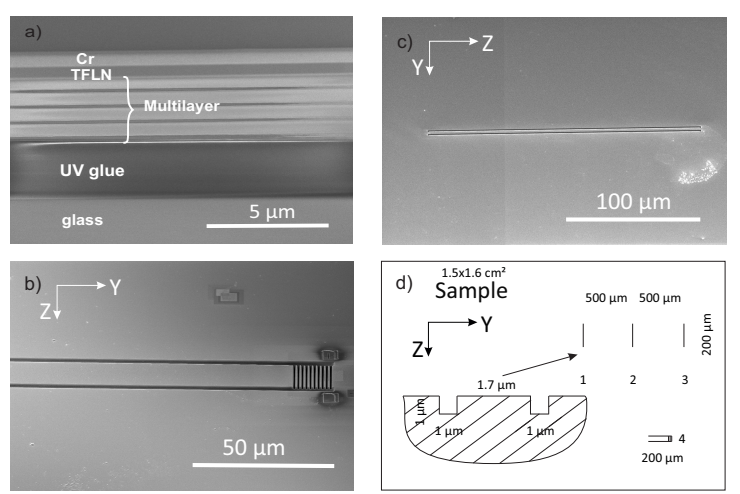

Fig. 4. (a) FIB-SEM (FEI Helios 600i) image of the sample crosssection, (b) FIB-SEM (FEI Helios 600i) image of the waveguide milled along $\mathrm{Y}$ axis of $\mathrm{LiNbO}_{3}$, (c) FIB-SEM (FEI Helios 600i) image of the scatterer, $(\mathrm{d})$ schematics of the scatterers on the sample. 


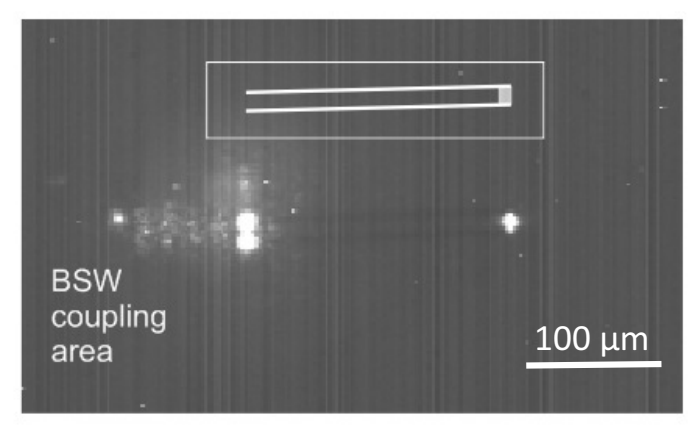

Fig. 5. The measured far-field image of the out-coupled BSW, which images the BSW coupling area, the entrance of the waveguide, and the grating out-coupler.

surface along the $\mathrm{Z}$ crystalline axis of the TFLN. The scatterers are defined by two grooves of $1 \mu \mathrm{m}$ width and $1 \mu \mathrm{m}$ depth at the distance of $1.7 \mu \mathrm{m}$ from each other. The SEM (FEI, Quanta 450W) image of a single scatterer is shown in Fig. 4c. Three scatterers at the distance of $0.5 \mathrm{~mm}$ from each other are milled by FIB (FEI Helios $600 \mathrm{i})$. The schematics of the scatterers' top view and cross section is shown in Fig. $4 \mathrm{~d}$.

\section{CHARACTERIZATION METHOD}

We use the Kretschmann configuration built on a glass prism (see Fig. 1) for the optical characterization. The light from a tunable laser (1480-1570nm) is focused on the sample through the prism. The illumination is flexible to adjust the size of the beam spot on the top surface of the multilayer chip, where the variable range of the spot size is from $10 \mu \mathrm{m}$ to $30 \mu \mathrm{m}$. The polarization of the incident beam is controlled by a Glan polarizer. On the other side of the prism, the reflected light is collected by an IR camera (camIR 1550, Applied Scintillation). The presence of absorption lines insures the coupling of the BSWs, as shown in Fig. 1b. The reflection dip is observed at an incident angle of $49^{\circ}$, which is very close to the theoretically calculated one $\left(48.5^{\circ}\right)$. The BSW excited in the TFLN layer propagates along the waveguide (Fig. 1a) or in a free 2D space at TFLN interface (Fig. 1c). BSW decouples through the grooves at the end of the waveguide (Fig. 1a) or through the scatterers (Fig. 1c). The out-coupled light is recorded by a microscopy imaging system, which combines a long-working distance objective lens (Nikon CF Plan, $\mathrm{NA}=0.35, \mathrm{x} 10$ and $\mathrm{x} 5$ - for grating decouplers on the waveguide and for scatterers respectively) and a digital IR camera (Xenix 135 XEVA-2232). The experimental setup for BSW wave-guiding study is shown in Fig. 1a. As an additional investigation the experimental setup for the BSW free space propagation is shown in Fig. 1c.

\section{RESULTS AND DISCUSSION}

The guided propagation of the BSW on the TFLN is experimentally demonstrated through a $200 \mu \mathrm{m}$ long multimode waveguide, see Fig. 5. The BSW is excited about $50 \mu \mathrm{m}$ away from the waveguide. In Fig.5, the two bright spots represent the scattering at the entrance of the waveguide. BSWs propagate through the waveguide and decouple by the gratings at the end of waveguide. The decoupled light is collected by the objective, which

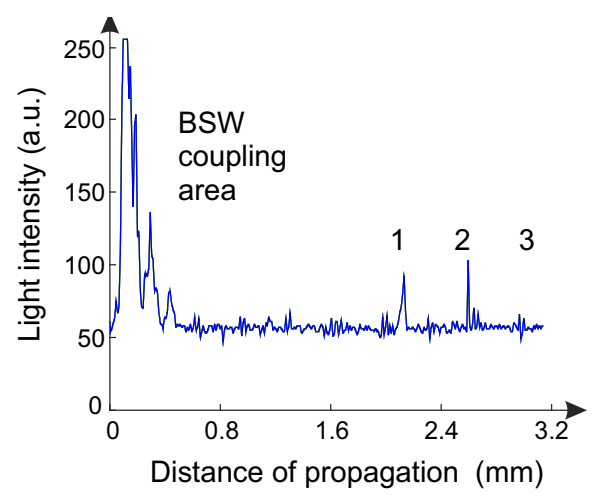

Fig. 6. Image profile for the BSW excited at $1.5 \mathrm{~mm}$ distance from the 1st scatterer.

can be referred as a bright spot at the end of the waveguide.

In order to estimate the BSW propagation on the $1 \mathrm{DPhC}+\mathrm{TFLN}$ the BSWs are launched along the $\mathrm{Y}$ axis in such a way that scatterer 1 , scatterer 2 and scatterer 3 perform as obstacles (see Fig. 1c and Fig. 4d). Light is coupled at $2 \mathrm{~mm}$ distance away from scatterer 1 . It partially scatters on each scatterer and continues propagating further. We can evidence from Fig. 6 that the BSW propagates until scatterer 2. Therefore, having known the distance between scatterers, about $0.5 \mathrm{~mm}$, we may estimate the BSW propagation still can be detected at the $3 \mathrm{~mm}$ distance away from the coupling area on the surface of 1DPhC with TFLN as a top layer. Despite of the use of a precise milling tool, such as FIB, the scatterers have some difference in dimensions: distance between grooves $-1.7 \pm 0.5 \mu \mathrm{m}$, width and depth of the grooves $-1.0 \pm 0.2 \mu \mathrm{m}$. This difference of the grooves size in the scatterers gives a difference in decoupling of light. Thus, we may observe a slightly stronger decoupling of light on the scatterers 2 in Fig. 6.

\section{CONCLUSIONS}

In this work, we present the propagation of BSWs along the ridge waveguide patterned into TFLN on the top of 1DPhC. The improved fabrication protocols allow a significant improvement of the surface quality of TFLN deposited on the top of 1DPhC. Therefore, the designed and fabricated multilayer stack (1DPhC+TFLN) couples BSWs at $1550 \mathrm{~nm}$ wavelength for integrated and nonlinear optics applications. We demonstrate experimentally the BSWs propagation along Y crystalline axis of $\mathrm{LiNbO}_{3}$ which can be potentially used for electro-optical modulation. Bloch surface wave's propagation of about $3 \mathrm{~mm}$ has been measured, for the presented design of 1DPhC with TFLN using a simple microscopy setup, at $1550 \mathrm{~nm}$ wavelength.

Fundings. This work is funded by the SMYLE program. It has been realized in the context of the Labex ACTION program (contract ANR-11-LABX-01-01). It is supported by the French RENATECH network and its FEMTO-ST technological facility.

Acknowledgments. The authors thank Raphaël Barbey for multilayer fabrication.

\section{REFERENCES}

1. P. Yeh, A. Yariv, A.Y. Cho, Appl. Phys. Lett. 32, 104-105 (1978). 
2. M. Liscidini,J.E. Sipe, Appl. Phys. Lett. 91, 253125 (2007).

3. E. Descrovi, F. Frascella, B. Sciacca, F. Geobaldo, L. Dominici, F. Michelotti, Appl. Phys. Lett. 91, 241109 (2007).

4. F. Giorgis, E. Descrovi, C. Summonte, L. Dominici, F. Michelotti, Opt. Express. 18, 8087-8093 (2010)

5. M. Liscidini, J.E. Sipe, J. Opt. Soc. Am. B 26, 279-289 (2009).

6. M. Liscidini, M. Galli, M. Patrini, R.W. Loo, M.C. Goh, C. Ricciardi, F. Giorgis, J.E. Sipe, Appl. Phys. Lett. 94, 043117 (2009).

7. R.G. Rodriguez, J.D. Ryckman, Y. Jiao, S.M. Weiss, Biosens. Bioelectron. 53, 486-493 (2014).

8. W. Kong, Z. Zheng, Y. Wan, S. Li, J. Liu, Sens. Actuator B-Chem. 193, 467-471 (2014)

9. A. Angelini, E. Enrico, N. De Leo, P. Munzert, L. Boarino, F. Michelotti, F. Giorgis, E. Descrovi New J. Phys. 15, 073002 (2013).

10. V.N. Konopsky, E.V. Alieva, Anal. Chem. 79, 4729-4735 (2007)

11. D.A Shilkin, E.V. Lyubin, I.V. Soboleva, A.A. Fedyanin, Opt. Lett. 40, 4883-4886 (2015)

12. F. Michelotti, B. Sciacca, L. Dominici, M. Quaglio, E. Descrovi, F. Giorgis, F. Geobaldo, Phys. Chem. Chem. Phys. 12, 502-506 (2010).

13. I.V. Soboleva, E. Descrovi, C. Summonte, A.A. Fedyanin, F. Giorgis, Appl. Phys. Lett. 94, 231122 (2009).

14. S. Pirotta, X.G. Xu, A. Delfan, S. Mysore, S. Maiti, G. Dacarro, M. Patrini, M. Galli, G. Guizzetti, D. Bajoni, J.E. Sipe, G.C. Walker, M. Liscidini, J. Phys. Chem. C 117, 6821-6825 (2013).

15. V.N. Konopsky, E.V. Alieva, S.Y. Alyatkin, A.A. Melnikov, S.V. Chekalin, V.M. Agranovich Light Sci Appl. 5, 16168 (2016).

16. V.N. Konopsky, D.V. Basmanov, E.V. Alieva, D.I. Dolgy, E.D. Olshansky, S.K. Sekatskii, G. Dietler, New J. Phys. 11, 063049 (2009).

17. D. Zhang, R. Wang, Y. Xiang, Y. Kuai, C. Kuang, R. Badugu,.. J.R Lakowicz, ACS Nano 11, 10446-10451 (2017).

18. R. Dubey, E. Barakat, M. Häyrinen, M. Roussey, S. Honkanen, M. Kuittinen, H.P. Herzig, J. Eur. Opt. Soc. 13, 1-9 (2017).

19. L. Yu, E. Barakat, T. Sfez, L. Hvozdara, J. Di Francesco, H.P. Herzig, LIGHT-SCI APPL 3, 124 (2014)

20. M.S. Kim, B.V. Lahijani, N. Descharmes, J. Straubel, F. Negredo C. Rockstuhl, M. Häyrinen, M. Kuittinen, M. Roussey, H.P. Herzig, ACS Photonics 4, 1477-1483 (2017).

21. E. Descrovi, T. Sfez, M. Quaglio, D. Brunazzo, L. Dominici, F. Michelotti, H.P. Herzig, O. Martin, F. Giorgis, Nano Lett. 10, 2087-2091 (2010).

22. M. Liscidini, D. Gerace, D. Sanvitto, D. Bajoni, Appl. Phys. Lett. 98 , 121118 (2011).

23. M. Liscidini, J. Opt. Soc. Am. B 29, 2103-2109 (2012).

24. L. Yu, "Near-field Imaging: Investigations on Bloch Surface Wave Based 2D Optics and the Development of Polarization-retrieved characterization" Ph.D. thesis, EPFL, (2013).

25. M. Menotti, M. Liscidini, J. Opt. Soc. Am. B 32, 431-438 (2015)

26. M. Zhang, H. Liu, H. Zhou, Z. Xiao, Opt Commun. 32, 479-482 (2018).

27. R. Dubey, E. Barakat, H.P. Herzig, "Bloch Surface Waves Based Platform for Integrated Optics," In Proceeding of the IEEE Photonics Conference, Reston, VA, USA, 4-8 October, 1092-8081 (2015).

28. R. Dubey, B.V. Lahijani, E. Barakat, M. Häyrinen, M. Roussey, M. Kuittinen, H.P. Herzig, Opt. Lett. 41, 4867-4870 (2016)

29. B.V. Lahijani, H.B. Ghavifekr, R. Dubey, M.S. Kim, I. Vartiainen, M. Roussey, H.P. Herzig, Opt. Lett. 42, 5137-5140 (2017).

30. R. Dubey, B.V. Lahijani, M.S. Kim, E. Barakat, M. Häyrinen, M. Roussey, M. Kuittinen, H.p. Herzig, "Near-field investigation of Bloch surface wave based 2D optical components" in Proceeding of the SPIE Photonics West 10106, San Francisco, CA, USA, 16 February, (2017).

31. R. Dubey, "Near-Field Characterization of Bloch Surface Waves Based 2D Optical Components," Ph.D. thesis, EPFL, (2017)

32. R. Dubey, B.V. Lahijani, M. Häyrinen, M. Roussey, M. Kuittinen, H.P. Herzig, Photonics Res. 5, 494-499 (2017)

33. R. Dubey, M. Marchena, B.V. Lahijani, M.S. Kim, V. Pruneri, H.P. Herzig, Appl. Sci. 8, 390 (2018)

34. V.N. Konopsky, T. Karakouz, E.V. Alieva, C. Vicario, S.K. Sekatskii, G. Dietler, Sensors 13, 2566-2578 (2013).

35. Y. Xiang, J. Guo, X. Dai, S. Wen, D. Tang, Opt. Express 22, 3054-3062 (2014).
36. S.K. Sekatskii, A. Smirnov, G. Dietler, M. Nur E Alam, M. Vasiliev, K. Alameh, Appl. Sci. 8, 248 (2018)

37. G. Lerario, D. Ballarini, L. Dominici, A. Fieramosca, A. Cannavale, M. Holwill, A. Kozikov, K.S. Novoselov, G. Gigli, Appl. Sci. 7, 1217 (2017).

38. T. Kovalevich, A. Ndao, M. Suarez, M. Häyrinen, M. Roussey, M. Kuittinen, T. Grosjean, M.P. Bernal, Opt. Lett. 41, 5616-5619 (2016).

39. L. Chen, R.M. Reano, Opt. Express 20, $4032-4038$ (2012).

40. W. Qiu, H. Lu, F.I. Baida, M.P. Bernal, Photon. Res. 5, 212-218 (2017)

41. C. Wang, M. Zhang, B. Stern, M. Lipson, M. Lončar, Opt. Express 26 1547-1555 (2018)

42. J. Wang, F. Bo, S. Wan, W. Li, F. Gao, J. Li, G. Zhang, J. Xu, Opt Express 23, 23072-23078 (2015)

43. T. Kovalevich, D. Belharet, L. Robert, M.S. Kim, H.P. Herzig, T. Grosjean, M.P. Bernal, Photon. Res. 5, 649-653 (2017).

44. V.N. Konopsky, New J. Phys. 12, 093006 (2010)

45. H. Okayama, "Lithium Niobate Electro-Optic Switching. Optical Switching," Springer, Boston, MA, 39-81 (2006).

46. A. Sinibaldi, N. Danz, E. Descrovi, P. Munzertb, U. Schulz, F. Sonntag, L. Dominici, F. Michelotti, Sens. Actuator B-Chem. 174, 292-298 (2012).

47. E. Kretschmann, H. Raether, Z. Naturforsch. 23, 2135-2136 (1968)

48. A.D. Bezpaly, V.M Shandarov, Phys. Procedia. 86, 166-169 (2017).

49. H. Han, L. Cai, H. Hu, Opt. Mater. 42, 47-51 (2015).

50. F. Lärmer, A. Schlip, "A Method of Anisotropically Etching Silicon," Licensed from Robert Bosch GmbH: US Patent No. 5,501,893 (1996).

51. M. Schaepkens, R.C.M. Bosch, T.E.F.M. Standaert, G.S. Oehrlein J.M. Cook, J. Vac. Sci. Technol. A 16, 2099-2107 (1998). 